\title{
The non-random walk of chiral magnetic charge carriers in artificial spin ice
}

SUBJECT AREAS: MAGNETIC PROPERTIES AND MATERIALS

METAMATERIALS

SPINTRONICS

STATISTICAL PHYSICS, THERMODYNAMICS AND NONLINEAR DYNAMICS

Received

25 October 2012

Accepted

14 January 2013

Published

13 February 2013

Correspondence and requests for materials should be addressed to W.R.B. (w.branford@ imperial.ac.uk)

* These authors contributed equally to this work.

\author{
K. Zeissler' ${ }^{1}$, S. K. Walton ${ }^{1 *}$, S. Ladak ${ }^{2}$, D. E. Read ${ }^{2}$, T. Tyliszczak ${ }^{3}$, L. F. Cohen ${ }^{1}$ \& W. R. Branford'
}

'Blackett Laboratory, Department of Physics, Imperial College, Prince Consort Road, South Kensington, London SW7 2AZ, ${ }^{2}$ School of Physics and Astronomy, Cardiff University, Queens Buildings, The Parade, Cardiff CF24 3AA, ${ }^{3}$ Advanced Light Source, Lawrence Berkeley National Laboratory, MS 6-2100 Berkeley, CA 94720, USA.

The flow of magnetic charge carriers (dubbed magnetic monopoles) through frustrated spin ice lattices, governed simply by Coulombic forces, represents a new direction in electromagnetism. Artificial spin ice nanoarrays realise this effect at room temperature, where the magnetic charge is carried by domain walls. Control of domain wall path is one important element of utilizing this new medium. By imaging the transit of domain walls across different connected 2D honeycomb structures we contribute an important aspect which will enable that control to be realized. Although apparently equivalent paths are presented to a domain wall as it approaches a Y-shaped vertex from a bar parallel to the field, we observe a stark non-random path distribution, which we attribute to the chirality of the magnetic charges. These observations are supported by detailed statistical modelling and micromagnetic simulations. The identification of chiral control to magnetic charge path selectivity invites analogy with spintronics.

pin Ice refers to a magnetic structure with a local ordering principle known as the ice rule, but no long range order $^{1-3}$. The absence of a single groundstate, first encountered in water ice ${ }^{4}$, has attracted great interest since it provides a strongly interacting system in which there are many energetically equivalent outcomes. Recent developments in nanofabrication have enabled the realisation of artificial spin ice materials composed of geometrically frustrated arrays of nanomagnets, which exhibit this local order with 'spins' on a lengthscale which allows direct imaging ${ }^{5-10}$. This class of materials includes nanomagnets arranged in both square ${ }^{11-14}$ and Kagome lattices $^{7,8,10,15-17}$, with either connected bars or individual islands. Traditional spin ice physics is governed by thermodynamics because the system is able to sample all possible states by thermal fluctuations of spins. In the artificial system the 'spins' are single domain ferromagnetic nanobars and the energy barrier to switching the macrosized spin is very large compared to the available thermal energy. Thus, in zero field, the underlying thermodynamic ground states can be completely suppressed by the kinetics of the system. However, hints at ordering temperatures of the order of $50 \mathrm{~K}$ have recently been observed ${ }^{18}$ in a field driven system where the kinetics are provided by the field.

The shape anisotropy of the nanobars forces the magnetisation to lie along the long axis. As such, the bars can be considered Ising macrospins, or dumbbells of magnetic charge, $\mathrm{q}$, the magnetisation of the bar divided by its length. The anisotropy forces the magnetisation of the bar to switch by nucleation and propagation of a domain wall, rather than coherent rotation. Each domain wall has a charge of $\pm 2 q$. In the honeycomb nanostructure three spins meet at each vertex and there is no magnetically charge neutral vertex configuration. The ice rule favours the six vertex configurations with total charge $Q= \pm q$ where either two of the three Ising like spins point into the vertex and one out, or two point out and one points in, the ice rules.

Violations to this ice rule in Kagome spin-ice arrays ${ }^{5-7,10}$, where all spins point in or out, are energetically unfavourable, but have been observed in magnetic reversals when either frozen disorder ${ }^{6,7}$ or magnetic charge carrier-carrier interactions ${ }^{5}$ play a role. These ice-rule-violating vertices with charge $Q= \pm 3 q$ are pure sources or sinks of magnetisation and are therefore described as magnetic monopole defects. In contrast the $Q= \pm q$ vertices carry both net magnetic charge and a net dipole moment and thus act as both sources and conduits of magnetic flux. The large energy cost associated with the formation of these monopole defects leads to particularly strong interbar correlations for 180 degree reversals where the magnetic field axis corresponds to the Ising axis of one of the subsets of bars. In this particular geometry switching only the subset of bars most strongly affected by the applied field would result in monopole defects at every vertex, a scenario at odds with the ice rule, which is not observed for connected bars. In honeycombs of well separated bars where the interbar coupling, and hence the ice rule, is much weaker, this multistage reversal is characteristic of the magnetic switching ${ }^{19}$. Similarly, if the field 
axis is rotated by 120 degrees after saturation along an Ising axis, stepwise magnetic reversal by subset of bars is readily observed in the connected honeycomb ${ }^{9}$ because it does not conflict with the ice rule.

A purely Coulombic model, in which the field required to switch a bar is described as the force required to separate a point charge of $\pm 2 \mathrm{q}$ (the ferromagnetic domain wall) from a oppositely charged vertex of charge $|Q|=q$ at an initial separation defined by the domain wall width, has been used successfully to describe the above discussed ice rule implications in artificial spin ice ${ }^{8,9,20}$. This point charge model predicts a large coercive field $\mathrm{H}_{\mathrm{C}}$ for nucleation of a domain wall at a vertex in an ice rule obeying state that is in good agreement with experimental observations $\mathrm{s}^{20}$ due to the large critical force $F$ needed to overcome magnetostatic attraction $\mathrm{F}=\mu_{0} \mathrm{H} .2 \mathrm{q} / \mathrm{w}$. However if a domain wall nucleated elsewhere approaches a vertex then it experiences instead a Coulombic repulsion between like charges and can escape much more easily. For this reason the switching tends to proceed by long cascades resulting from the nucleation of a few walls which then each propagate right across the lattice (figure 1a).
If the reversal is driven by a field parallel to one subset of bars then at every other vertex the cascade reaches decision points that have two nominally equivalent outcomes in the point charge model (figure $1 \mathrm{~b}$ ). Hence in artificial spin ice, long chains comprising of bars reversing together were found to be a signature of the $180^{\circ}$ magnetic reversal, irrespective of whether one discusses connected or unconnected structures ${ }^{8,10}$. In the connected artificial spin ices case, studied in this publication, these long chains can be readily observed. However our observation is that their propagation did not exclusively obey a random walk pattern which would follow naturally from the point like charge arguments discussed in the recent advances in the artificial spin ice community ${ }^{8,9,20}$. Figure 1a shows these long unidirectional chains; if at any vertex the probability of a domain wall propagating through one of the two identical $\pm 60^{\circ}$ diagonal bar is $50 / 50$ then chains where 9 subsequent $+60^{\circ}$ diagonal bars have switched are unlikely to occur.

Considering the magnetic moment distribution in a domain wall can shed light on this phenomenon. Domain walls in magnetic nanowires are chiral objects, and this chirality breaks the symmetry at the
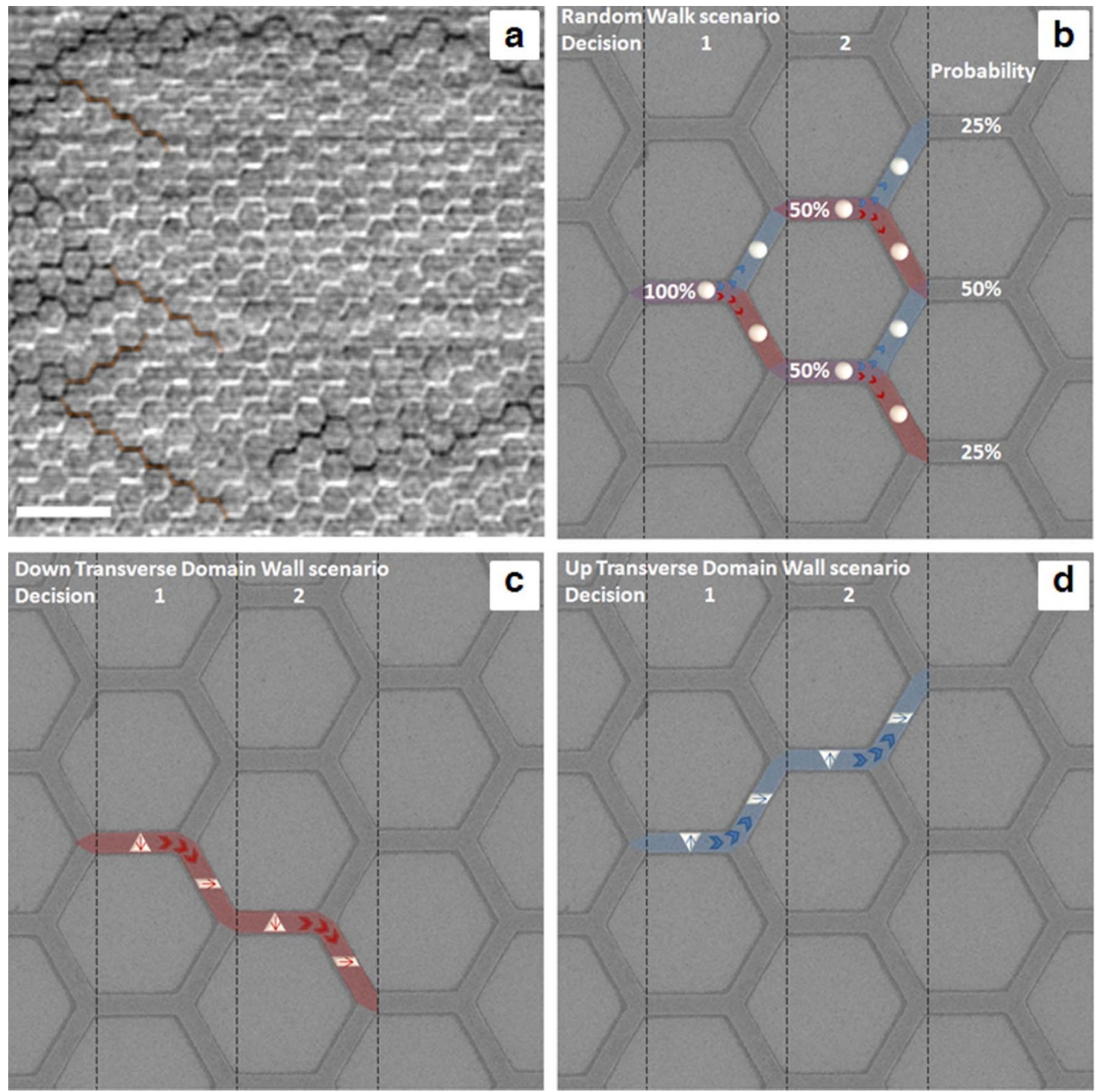

Figure $1 \mid$ Domain wall movement through permalloy honeycomb artificial spin ice. (a) XMCD image shows long unidirectional chains of switched bars (orange). Scale bar is $5 \mu \mathrm{m}$. (b). Schematics showing the random walk scenario which is expected if domain walls are portrayed as point-like magnetic charges moving through the structure. At each junction there is a 50/50 chance of taking the $\pm 60^{\circ}$ bar. However when one considers the true shape of the domain walls, in this case transverse domain walls, then the non uniform magnetic moment distribution will have an effect on the propagation path. (c)-(d) Schematics illustrating the down and up chiral transverse domain wall cases respectively. 
vertex, giving rise to the possibility of selectivity. A similar type of selectivity was recently observed in cross-shaped nanowire vertices ${ }^{21}$. The magnetic domain wall type and structure depends on the bar dimensions; in the permalloy artificial spin ice studied in this publication, transverse domain walls are supported ${ }^{22}$. In this study we discuss the effect of the chirality of transverse domain walls on the propagation direction chosen at each vertex in a $180^{\circ}$ reversal scenario. The chirality describes the sense of rotation of the magnetization within the domain wall. The internal structure of transverse walls has characteristic triangular shape and transverse magnetic moment, which is illustrated for walls with down and up chirality in figures 1c and 1d respectively. We argue that this additional consideration results in a selectivity governed by the domain wall chirality, leading to the observation of non-random path distribution.

We clearly observe a non-random path distribution, even when the separation between junctions appears to be greater than the expected Walker length. The implication of the work is that the chirality of the magnetic charge carriers controls their propagation decisions providing a distinctive element of control in magnetic charge propagation and inviting analogies with spintronics.

\section{Results}

The correlations between propagation selections during the magnetic reversal were studied in connected permalloy artificial spin ice. Individual switching chains were identified in step wise reversed artificial spin ice arrays of thickness $18 \mathrm{~nm}$ comprising of bars of length $1 \mu \mathrm{m}$ and $2 \mu \mathrm{m}$. Figure 2 a shows a typical honeycomb array in mid transition, white and black XMCD contrast identifies magnetisation along the positive or negative $\mathrm{x}$ axis respectively. See supplementary information for movies of the large area scans ( $\mathrm{S} 1$ for the switching of the $1 \mu \mathrm{m}$ array and S2 for the $2 \mu \mathrm{m}$ data). The origin of individual chains was defined as $(0,0)$ and the y displacement was tracked until the chain terminated or branched out (see figure $2 \mathrm{~b}$ ). The y displacement of all identified chains is summarized in figure $3 \mathrm{a}$ for the $1 \mu \mathrm{m}$ array and figure $3 \mathrm{~b}$ for the $2 \mu \mathrm{m}$ array. The strong difference of the observed distribution compared to the randomwalk distribution is illustrated in figures $3 c$ and $3 d$ where the raw data has been normalised by the expected (binomial) random walk distribution. If the random walk correctly describes our system and there is a $50: 50$ probability at each decision point, this normalisation would be expected to give a flat distribution at each decision point (chain length). Instead we see a very strong excess at the edges where $|y|$ is maximum.

Breaking down the chains into two consecutive decisions leads to 3 possible y displacements with coordinates $(2,0),,(2,2)(2,-2)$. The random walk probabilities of occurrence associated with these are $1 / 2$, $1 / 4$ and $1 / 4$ respectively. The $(2,2)$ and $(2,-2)$ cases are associated with long unidirectional chains. We find that $72.5 \%$ of the twodecision correlators terminated at $(2,2)$ or $(2,-2)$ in the artificial spin ice with a bar length of $1 \mu \mathrm{m}$, in comparison with $58.0 \%$ in the $2 \mu \mathrm{m}$ artificial spin ice (Table 1 ). If the switching path were entirely random, we would expect there to be an equal probability for the domain wall to remain in the same direction of propagation or to change propagation direction, so a binomial (random walk) distribution would be seen. An exact binomial test for goodness of fit was performed for both the $1 \mu \mathrm{m}$ and $2 \mu \mathrm{m}$ artificial spin ices. In the $1 \mu \mathrm{m}$ artificial spin ice the domain walls retained their direction in 124 out of 171 events, which deviates from a random walk scenario with a one-tailed confidence interval of $5 \%$ with a p-value of $1.7^{*} 10^{-9} 24$. In the $2 \mu \mathrm{m}$ artificial spin ice 76 out of 131 events retained their direction deviates from a random walk scenario with a one-tailed confidence interval of $5 \%$ with a p-value 0.04 .

Micromagnetic simulations were performed on the field-driven transit of a domain wall through a single vertex at $0 \mathrm{~K}$ using the OOMMF software ${ }^{22}$. In the simulation perfect selectivity was observed in the transverse domain wall regime. A domain wall which propagates through the horizontal bar was seen to result in the switching of the $+60^{\circ}$ or $-60^{\circ}$ diagonal bar depending on whether an up (figure $4 \mathrm{a}-\mathrm{b}$ ) or down (figure $4 \mathrm{c}-\mathrm{d}$ ) chiral transverse wall was injected. During their transit through these diagonal bars they are distorted and the Zeeman force aligns the moment of the walls with the external field (figure $4 \mathrm{~b}-\mathrm{d}$ ) effectively resetting the chirality of the transverse domain wall between each horizontal bar. However figure $4 \mathrm{e}-\mathrm{h}$ shows that the chirality depinning from the vertex next in line depends on whether the domain wall arrives from the lower branch or the upper branch. A domain wall arriving from the lower branch depins as an up transverse domain wall (figure $4 \mathrm{e}-\mathrm{f}$ ) and a domain wall arriving from the upper branch depins as a down transverse domain (figure $4 \mathrm{~g}-\mathrm{h}$ ). In this way the chirality of the bar entering the second bar parallel to the field is determined by the chirality of the wall that exited the first parallel bar. Thus, chiral information can be passed through the array as the domain wall continues to
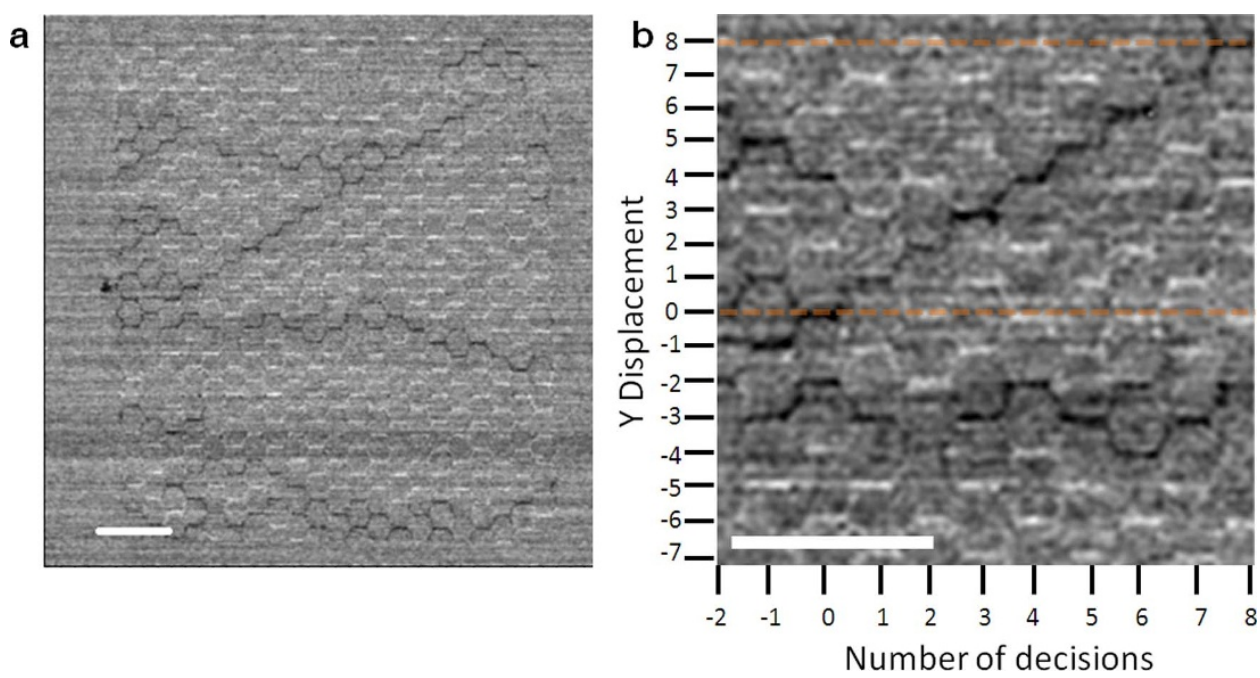

Figure $2 \mid$ Mapping the path of individual carriers. (a) A typical STXM image for a partially switched $18 \mathrm{~nm}$ thick artificial spin ice with $100 \mathrm{~nm}$ wide and $1 \mu \mathrm{m}$ long bars. Scale bar $5 \mu \mathrm{m}$. The array was initially saturated along $\mathrm{x}$ and then a $-9.25 \mathrm{mT}$ field was applied in the negative $\mathrm{x}$ direction. White contrast indicates magnetisation in the positive $\mathrm{x}$ direction, black contrast indicates magnetisation in the negative $\mathrm{x}$ direction. (b) A magnified region of (a) illustrating the counting method used. Scale bar $5 \mu \mathrm{m}$. The beginning of the chain, the first identifiable horizontal bar, is defined as $(0,0)$. The coordinates of each other bar in the chain is defined with respect to this origin as shown on the axes. 
a

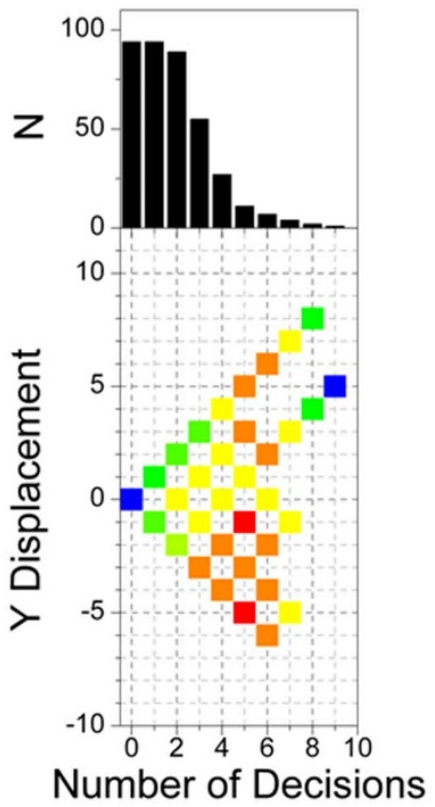

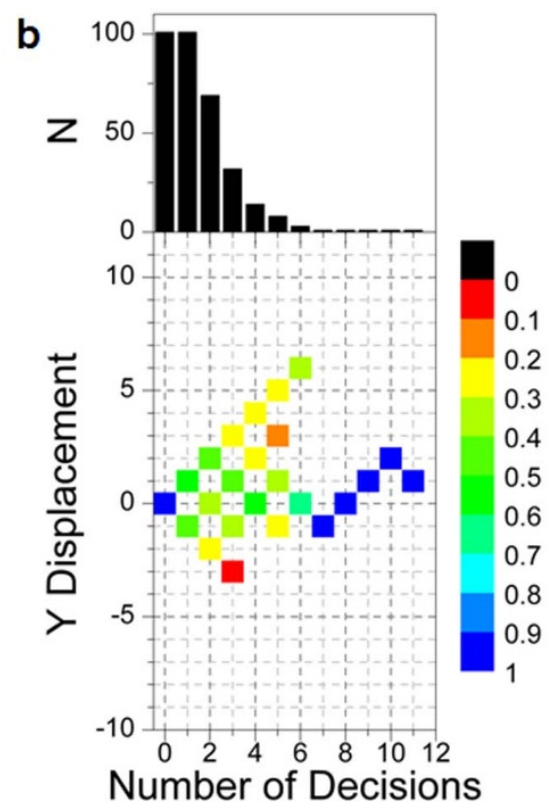

Number of Decisions
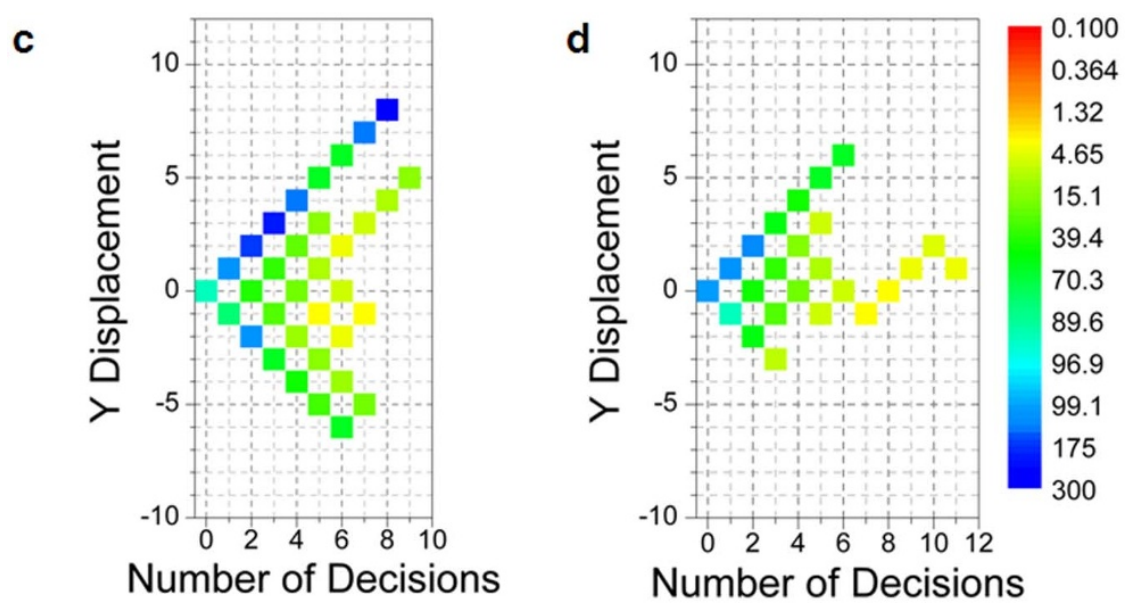

Figure 3 Distribution of observed magnetic charge paths during 180 degree magnetic reversals in $18 \mathrm{~nm}$ thick honeycomb arrays of bar length (a) $1 \mu \mathrm{m}$ and (b) $2 \mu \mathrm{m}$. The black histograms show the total number of chains observed $(N)$ after a given number of decisions. The displacement of the path of the isolated charge carrier in the direction perpendicular to the applied field is the y-coordinate which is recorded as a function of the chain length or number of decisions (x-coordinate) as defined in the text. At each decision point $\Delta y= \pm 1, \mathrm{~N}_{\mathrm{y}}$, is the number of observations of chains at each particular $(\mathrm{x}, \mathrm{y})$ coordinate, and $\left(\mathrm{P}_{\mathrm{RW}}\right)$ is the probability of reaching that $(\mathrm{x}, \mathrm{y})$ position in a random walk scenario where at each decision point the two outcomes $\Delta y=+1$ and $\Delta y=-1$ each have a probability of 0.50 . The colour coded (see legend) plots in (a) and (b) show $N_{y}$ normalised by $N$. (c) and (d) show the $\mathrm{N}_{\mathrm{y}}$ data colour-coded as $\mathrm{N}_{\mathrm{y}} / \mathrm{P}_{\mathrm{RW}}$ (see legend). A full breakdown of the raw data contained in this figure is contained in supplementary table $\mathrm{S} 3$.

propagate, even though the chirality of the wall is reset in the intervening bar at \pm 60 degrees to the field. This leads to domain wall propagation paths that deviate from the random walk model. More specifically, this could explain why there is an unexpectedly high occurrence of long unidirectional chains observed during $180^{\circ}$ reversals observed in this paper.

\section{Discussion}

Let us consider field misalignment as a source of asymmetry in the propagation. The data also showed that in total, more domain walls took an upward branch than a downward branch, which was more pronounced in the $18 \mathrm{~nm}$ data. Our data comes from the same series of runs on arrays grown on the same membrane without changing the sample and magnet mounting, which were aligned by eye with an error of less than five degrees. This overall asymmetry could be related to field misalignment, or even something systematic in the sample fabrication that favours asymmetric domain wall nucleation (no such asymmetry is apparent in our images). However in our data (Table 1) we see much greater intensity than expected from a random walk model in both the $+y$ and $-y$ extremities for a single sample alignment, a typical illustration of this can be found in figure $2 a$. This cannot be explained by field misalignment. The magnetisation versus external field is plotted in figure $5 \mathrm{a}$ and shows a sharp reversal without any plateaux. This also indicates that the field misalignment is not the source of our selectivity and systematic fabrication imperfections do not dominate the reversal. Note that in our (zero temperature) simulations we are unable to introduce a partial bias with field misalignment, we see no effect at small angles, and perfect selectivity at greater misalignments, with the crossover at about seven degrees. The combination of field misalignment with temperature or quenched disorder may be able to produce a biased random walk distribution, and is a possible explanation for the overall asymmetry between up and down in our statistics. However we stress that both the $+\mathrm{y}$ and $-\mathrm{y}$ extremities are strongly favoured over the random-walk 
Table 1 | Summation of the change in $y$-displacement $(\Delta y)$ of observed consecutive decisions in a transit in the $x$-direction $(\Delta x$ $=2$ ). The random walk model (null hypothesis) assumes that there is no overall preference for $\Delta y= \pm 1$ in any decision and therefore that there should be no correlation between the first and second decisions. The observed populations at $(\Delta x, \Delta y)=(2, \pm 2)$ compared to $(\Delta x, \Delta y)=(2,0)$ were tested against the null hypothesis of no correlation (where the expected $([0.25+0.25]: 0.50=$ $0.5: 0.5)$ distribution is illustrated in figure $1 \mathrm{~b}$ ) using a 1 -tailed binomial test. The exact binomial one tailed test quantifies the probability that random walk model over our number of total observations would produce a data set as far, or further, from the expected $50: 50$ ratio. The $p$-value should be less than the significance level, which is typically taken to be 0.05

\begin{tabular}{lcc} 
& \multicolumn{3}{c}{ Artificial Spin Ice } \\
\cline { 2 - 3 }$(\Delta \mathrm{x}, \Delta \mathrm{y})$ & $1 \mu \mathrm{m}$ & $2 \mu \mathrm{m}$ \\
\hline$(2,2)$ & 68 & 56 \\
$(2,0)$ & 47 & 55 \\
$(2,-2)$ & 56 & 20 \\
$(2, \pm 2)$ & 124 & 76 \\
$(2,0)$ & 47 & 55 \\
Total & 171 & 131 \\
1-Tailed Test & $1.7^{*} 10^{-9}$ & 0.04 \\
\hline
\end{tabular}

model in data sets collected from a single sample mounting, and a bias to both extremes cannot be simply a matter of alignment.

Now let us consider the mechanism by which the chirality breaks the symmetry and causes directional selectivity. A closer look at the magnetic configuration near the vertex enables us to understand which chirality favours which path. As the domain wall approaches the vertex it affects the local magnetisation in and around the vertex. In both transverse cases a ' $C$ '-like state (an intermediate state of low exchange energy cost seen in nanodiscs of certain dimension ${ }^{25}$ ) is stabilized near the vertex but the location of the $\mathrm{C}$-like state depends on the chirality. In the up situation, the C-like state is stabilized over the upper and horizontal bars, which is an equivalent situation to the nucleation of a domain wall in the upper branch (figure 4a). Conversely, in the down chirality situation, the C-like state is stabilized over the lower and horizontal branches, equivalent to the nucleation of a domain wall in the lower branch (figure 4c). As the field is increased, it is these domain walls that propagate through the array, causing directional selectivity.

Despite these transverse domain wall dependent switching rules, the reversal is still a potentially stochastic process as the domain wall travelling through a nanowire can undergo Walker breakdown. Walker breakdown refers to the change of domain wall chirality via intermediate antivortex states when the domain wall is forced to propagate down a wire due to an externally applied magnetic field ${ }^{26,27}$. There are three identifiable regimes as a function of field in the Walker breakdown mechanism. Below the Walker field $\mathrm{H}_{\mathrm{W}}(\mathrm{H}$ $<\mathrm{H}_{\mathrm{W}}$ ) the transverse domain wall propagates down the wire without deformation, just above $\mathrm{H}_{\mathrm{W}}\left(1<\mathrm{H} / \mathrm{H}_{\mathrm{W}}<5.5\right)$ the chirality periodically changes from up to down transverse domain walls via the formation of an antivortex domain wall and at high fields $\left(\mathrm{H} / \mathrm{H}_{\mathrm{W}}>\right.$ 5.5) the transverse domain wall chirality changes via multivortex and multiantivortex states $^{28,29}$. Theoretical calculations predict the Walker Breakdown field to be $\mathrm{H}_{\mathrm{W}} \sim 6.5$ Oe at zero temperature for perfectly smooth wires, $100 \mathrm{~nm}$ wide and $18 \mathrm{~nm}$ thick $^{30}$, which would place our system well into region 3 . The fidelity length, the length up to which the chirality is preserved, is predicted to be $0.5 \mu \mathrm{m}^{29}$ for simulations carried out at $0 \mathrm{~K}$. Room temperature experiments on permalloy wires ( $90 \mathrm{~nm}$ wide, $12 \mathrm{~nm}$ thick) measured a fidelity length of $\sim 0.4 \mu \mathrm{m}$ in a 100 Oe field $^{31}$. However edge

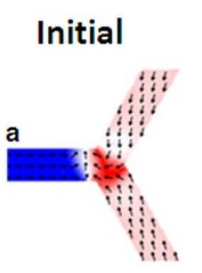

Final
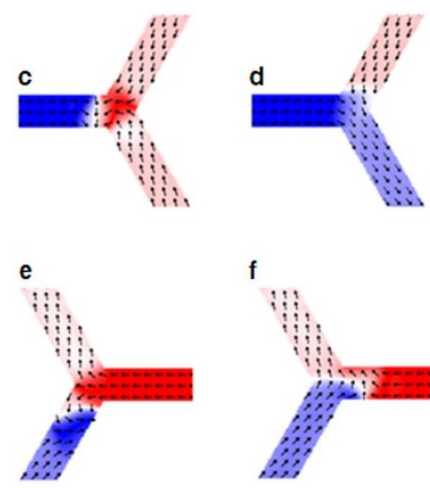

f
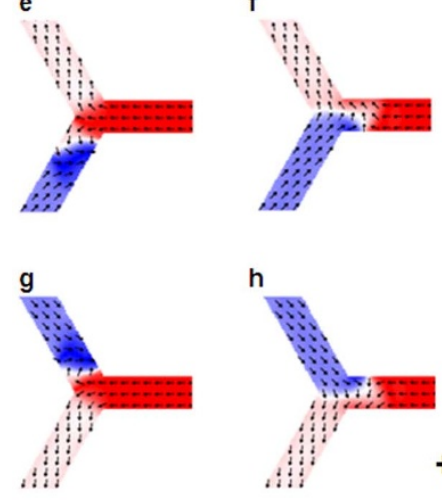

h

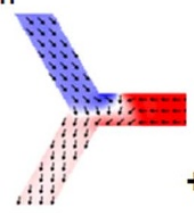

H
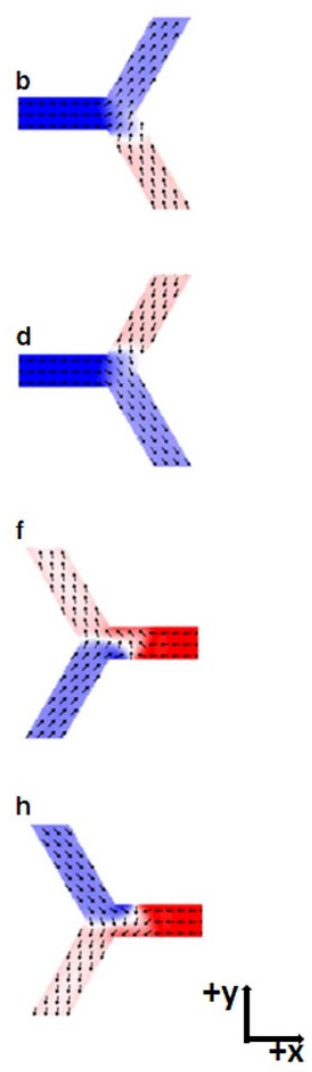

Figure $4 \mid$ Simulation of domain wall movement through vertex. OOMMF simulations of a single honeycomb spin ice vertex with a thickness of $18 \mathrm{~nm}$ and width $100 \mathrm{~nm}$. The switching, which is mediated by an artificially injected transverse domain wall moving through the vertex, is driven by an applied field in the $+\mathrm{x}$ direction after saturation in the - $\mathrm{x}$-direction. The initial and final states show the magnetic structure before and after the wall has crossed the vertex. (a) - (b) Switching via a transverse domain wall with up chirality and (c) - (d) down chirality. (e) - (f) Shows the switching from the lower diagonal bar and the formation of an up chirality transverse domain wall. (g) - (h) Shows the switching from the upper diagonal bar and the formation of a down chirality transverse domain wall.

roughness can increase $\mathrm{H}_{\mathrm{W}}$, suppressing the onset of Walker breakdown $^{32}$ and our observations suggest that this is the case. With a bar length of $1 \mu \mathrm{m}$ we expect to see some Walker breakdown, manifested in the washing out of the selectivity produced by the chirality rule. However as $72.5 \%$ selectivity remains in our data, our edge roughness might be sufficiently suitable for a majority of our bars suppressing the Walker breakdown. Selectivity decreases severely for the artificial spin ice with bar length of $2 \mu \mathrm{m}$ emphasising the influence of Walker breakdown.

Statistically insignificant numbers of long chains of switched bars were seen in our arrays (see figure $5 c$ ). Long chains can only be tracked at early stages in the reversal since later on, there are many domain walls in the array and it is hard to infer which domain wall switches which bar. A simulation was performed to demonstrate the differences in the distributions of switched bars expected from both a pseudorandom walk and a pseudorandom biased walk (see figure $5 \mathrm{~b}$ ). In the latter, the first pseudorandom decision is unbiased $(50: 50)$ and each subsequent decision is pseudorandom with a bias of $72.5: 27.5$, for example if the first decision results in a switched bar at $(1,1)$ then there is a $72.5 \%$ likelihood that the next decision results 

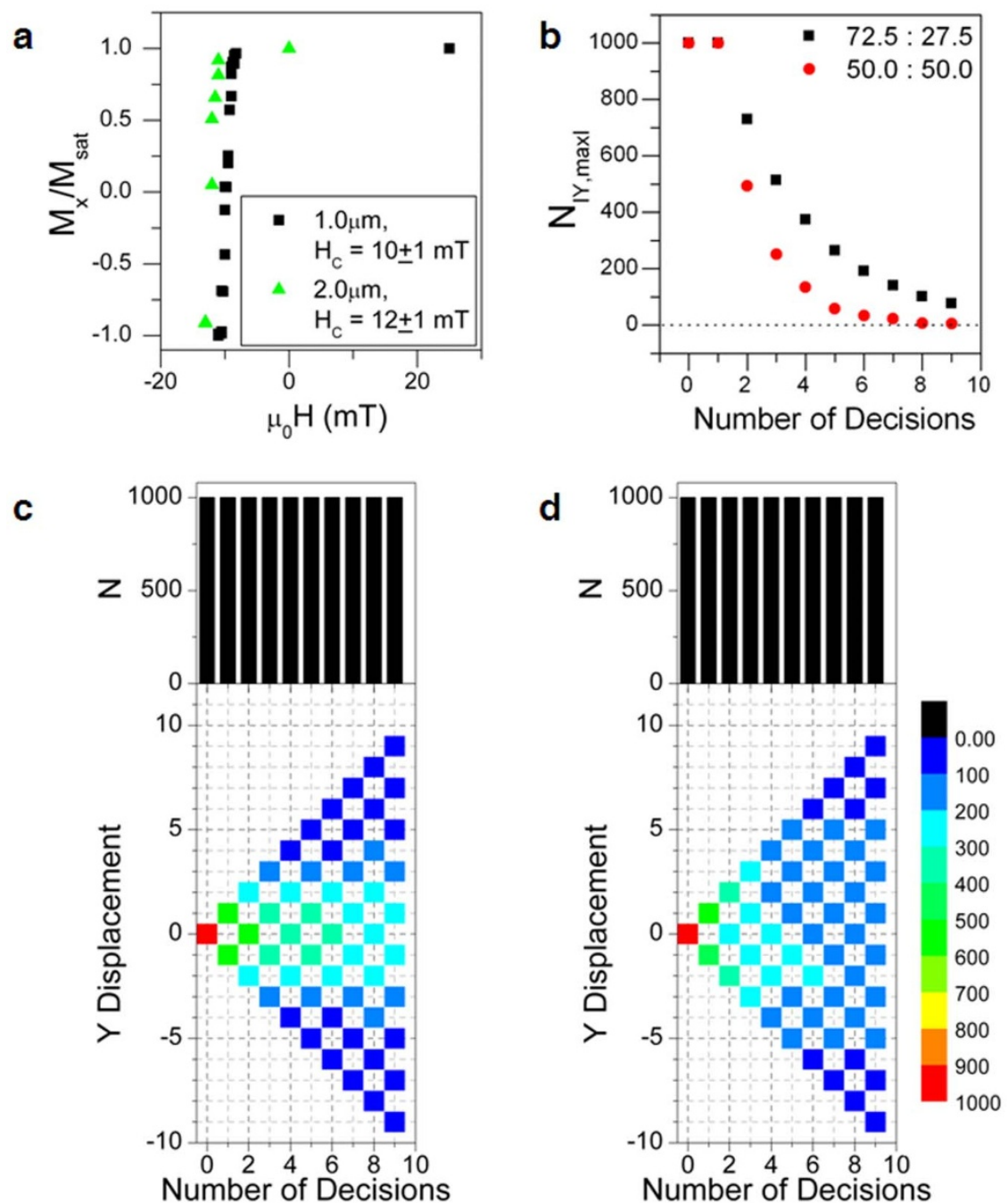

Figure 5 Experimental magnetisation change and pseudorandom walk scenario (a) Room temperature magnetisation reversal, after initial saturation in the positive $\mathbf{x}$ direction, of the artificial spin ice arrays. The normalized magnetisation is calculated by summing the number of bars with a particular STXM magnetic contrast (figure 2) normalised by the total number of bars. Sharp magnetic reversal without any plateaus indicates that there is no field misalignment and that any fabrication defects have an insignificant influence on the switching. (b) Expected number of observations at the outer most possible path ( $\left.\mathrm{N}_{|\mathrm{Ymax}|}\right)$ after 1000 random walks and biased random walks. The colour-coded (see legend) full path distribution is shown in (c) for random walks and (d) biased random walks. The black histograms show the total number of chains simulated $(N)$ after a given number of decisions. The biased random walk model assumes that there is no overall preference for $\Delta y= \pm 1$, but that a subsequent decision is correlated with the immediately preceding decision. We used the apparent bias factor from the $1 \mu \mathrm{m}$ experimental two decision correlation data (see Table 1$)$ of $72.5: 27.5$.

in a switched bar at $(2,2)$. The addition of a bias results in a greater population of switched bars at the extremities and a reduced population of switched bars around a Y displacement of zero compared to the random walk scenario; witnessing a unidirectional chain of 8 decisions is nearly 20 times more likely in the biased situation than it is in the random walk. In order to improve the statistical viability of the study by witnessing more long chain events, it would be advantageous to have larger arrays or to restrict the number of domain walls in the arrays, perhaps via domain wall nucleation suppression at the edges of the arrays.

The long isolated chains that we observe (figure laonly appear in the early stages of the reversal, and our number of observations decreases rapidly with increasing chain length, as shown in the histograms in figure $3 \mathrm{a}$ and $\mathrm{b}$. Thus it is challenging to obtain statistically significant numbers of long chain observations. For this reason we have grouped together all observations of two consecutive decisions, regardless of chain length, for our statistical analysis in Table 1. Simulations of 1000 random walks undergoing nine consecutive decisions were performed for both an unbiased walk and for a biased random walk (figure $5 \mathrm{c}$ and $\mathrm{d}$ respectively). The biased random walk model assumes that there is no overall preference for $\Delta y= \pm 1$, but that a subsequent decision is correlated with the immediately preceding decision. We used the apparent bias factor from the $1 \mu \mathrm{m}$ experimental two decision correlation data (see Table 1) of $72.5: 27.5$. The difference in the expected number of observations at the outer most possible path in both positive $y$ displacement and negative y displacement $\left(\mathrm{N}_{|\mathrm{Ymax}|}\right)$ is shown in figure $5 \mathrm{~b}$. Witnessing a unidirectional chain after nine consecutive path decisions is nearly 20 times more likely for a biased walk in our model. In order to improve the statistical viability of the study by witnessing more long chain events, it would be advantageous to have larger arrays or to restrict the number of domain walls in the arrays, perhaps via domain wall nucleation suppression at the edges of the arrays.

In summary we find that as a domain wall approaches a complex magnetic vertex, the chirality of the wall can break the apparent symmetry of the system, leading to non-random propagation. Our structures have bars with length of order of the fidelity length associated with Walker breakdown, so we observe biased random walk 
behaviour with the degree of bias controlled by the chance of loss of chiral information. This suggests that if a domain wall can be injected with a specific chirality and the bar length is significantly less than the fidelity length then its path through the two-dimensional array can be controlled. Since the results can be directly imaged, the structures provide an interesting playground for testing physics associated with spin-orbit effects such as the spin-Hall effect, and could potentially lead to new architectures for parallel computation.

\section{Methods}

Artificial 2D kagome arrays were structured from $\mathrm{Ni}_{81} \mathrm{Fe}_{19}$ using electron beam lithography, thermal evaporation and a lift off processing technique. The magnetisation reversal was studied using room temperature Scanning Transmission X-ray Microscopy (STXM) carried out on beamline 11.02 at the Advanced Light Source (Berkeley, CA, USA). The sample was mounted between pole pieces of an electromagnet allowing the application of an in-plane field of $\pm 250 \mathrm{mT}$ in situ. The chamber was pumped down to a pressure of approximately $100 \mathrm{mTorr}$ before filling with $\mathrm{He}$ gas. Elliptically polarized $\mathrm{x}$-rays were provided by an undulator beamline after which they were focused to a spot size of approximately $30 \mathrm{~nm}$ using a Fresnel zone plate. The in-plane component of the magnetisation was probed using the $\mathrm{x}$-ray magnetic circular dichroism (XMCD) effect by mounting the sample and the electromagnet approximately 30 degrees with respect to the $\mathrm{x}$-ray propagation vector. In order to study the switching of the array, the sample was first saturated in the positive field direction, and then small field increments were applied in the negative field direction.

Micromagnetic Simulations were performed using the Object Oriented Micromagnetic Framework (OOMMF) software ${ }^{23}$. The simulations are effectively at absolute zero temperature. Mesh sizes of [ $5 \mathrm{~nm}, 5 \mathrm{~nm}, 6 \mathrm{~nm}$ ] for $18 \mathrm{~nm}$ thick bars and [ $5 \mathrm{~nm}, 5 \mathrm{~nm}, 9 \mathrm{~nm}$ ] for $36 \mathrm{~nm}$ thick bars were used. The exchange constant and saturation magnetisation were taken to be $13 * 10^{-12} \mathrm{Jm}^{-1}$ and $800 \mathrm{kAm}^{-1}$ respectively. The domain walls were introduced into the OOMMF simulation and their chirality preconditioned via a colour map. In order to prevent switching via domain wall nucleation, a stitching field was initialized and imposed on the edges of the bars. An external magnetic field was applied to a single vertex in the positive $\mathrm{x}$ direction after a saturating magnetic field was applied in the negative $\mathrm{x}$ direction.

1. Bramwell, S. T. \& Gingras, M. J. P. Spin ice state in frustrated magnetic pyrochlore materials. Science 294, 1495-1501 (2001).

2. Harris, M. J., Bramwell, S. T., Holdsworth, P. C. W. \& Champion, J. D. M. Liquidgas critical behavior in a frustrated pyrochlore ferromagnet. Phys. Rev. Lett. 81, 4496-4499 (1998).

3. Ramirez, A. P., Hayashi, A., Cava, R. J., Siddharthan, R. \& Shastry, B. S. Zero-point entropy in 'spin ice'. Nature 399, 333-335 (1999).

4. Pauling, L. The structure and entropy of ice and of other crystals with some randomness of atomic arrangement. J. Am. Chem. Soc 57, 2680-2684 (1935)

5. Ladak, S., Read, D., Tyliszczak, T., Branford, W. R. \& Cohen, L. F. Monopole defects and magnetic Coulomb blockade. New Journal of Physics 13, 023023 (2011).

6. Ladak, S., Read, D. E., Branford, W. R. \& Cohen, L. F. Direct observation and control of magnetic monopole defects in an artificial spin-ice material. New Journal of Physics 13, 063032 (2011).

7. Ladak, S., Read, D. E., Perkins, G. K., Cohen, L. F. \& Branford, W. R. Direct observation of magnetic monopole defects in an artificial spin-ice system. Nature Physics 6, 359-363 (2010).

8. Daunheimer, S. A., Petrova, O., Tchernyshyov, O. \& Cumings, J. Reducing Disorder in Artificial Kagome Ice. Phys. Rev. Lett. 107, 167201 (2011).

9. Mellado, P., Petrova, O., Shen, Y. C. \& Tchernyshyov, O. Dynamics of Magnetic Charges in Artificial Spin Ice. Phys. Rev. Lett. 105, 187206 (2010).

10. Mengotti, E., Heyderman, L. J., Rodriguez, A. F., Nolting, F., Hugli, R. V. \& Braun, H. B. Real-space observation of emergent magnetic monopoles and associated Dirac strings in artificial kagome spin ice. Nature Physics 7, 68-74 (2011).

11. Mol, L. A., Silva, R. L., Silva, R. C., Pereira, A. R., Moura-Melo, W. A. \& Costa, B. V. Magnetic monopole and string excitations in two-dimensional spin ice. J. Appl. Phys. 106, 063913 (2009).

12. Morgan, J. P., Stein, A., Langridge, S. \& Marrows, C. H. Thermal ground-state ordering and elementary excitations in artificial magnetic square ice. Nature Physics 7, 75-79 (2011).

13. Morgan, J. P., Stein, A., Langridge, S. \& Marrows, C. H. Magnetic reversal of an artificial square ice: dipolar correlation and charge ordering. New Journal of Physics 13, 105002 (2011).

14. Wang, R. F., Nisoli, C., Freitas, R. S., Li, J., McConville, W., Cooley, B. J., Lund, M. S., Samarth, N., Leighton, C., Crespi, V. H. \& Schiffer, P. Artificial 'spin ice' in a geometrically frustrated lattice of nanoscale ferromagnetic islands. Nature 439, 303-306 (2006).

15. Moller, G. \& Moessner, R. Magnetic multipole analysis of kagome and artificial spin-ice dipolar arrays. Phys. Rev. B 80, 140409 (2009).

16. Qi, Y., Brintlinger, T. \& Cumings, J. Direct observation of the ice rule in an artificial kagome spin ice. Phys. Rev. B 77, 094418 (2008).

17. Rougemaille, N., Montaigne, F., Canals, B., Duluard, A., Lacour, D., Hehn, M., Belkhou, R., Fruchart, O., El Moussaoui, S., Bendounan, A. \& F, M. Artificial Kagome Arrays of Nanomagnets: A Frozen Dipolar Spin Ice. Phys. Rev. Lett. 106, 057209 (2011).

18. Branford, W. R., Ladak, S., Read, D. E., Zeissler, K. \& Cohen, L. F. Emerging Chirality in Artificial Spin Ice. Science 335, 1597-1600 (2012).

19. Schumann, A., Sothmann, B., Szary, P. \& Zabel, H. Charge ordering of magnetic dipoles in artificial honeycomb patterns. Appl. Phys. Lett. 97, 022509 (2010).

20. Tchernyshyov, O. Magnetic Monopoles - No longer on thin ice. Nature Physics 6, 323-324 (2010).

21. O’Brien, L., Beguivin, A., Petit, D., Fernandez-Pacheco, A., Read, D. \& Cowburn, R. P. Domain Wall interactions at a cross shaped vertex. Philos. Trans. R. Soc. Lond. Ser. A-Math. Phys. Eng. Sci. 370, 5794 (2012).

22. Ladak, S., Walton, S. K., Zeissler, K., Tyliszczak, T., Read, D. E., Branford, W. R. \& Cohen, L. F. Disorder-independent control of magnetic monopole defect population in artificial spin-ice honeycombs. New Journal of Physics 14, 045010 (2012).

23. http://math.nist.gov/oommf. (28 Sept 2012 update).

24. Iversen, G. R. Bayesian Statistical Inference. Quantitative Applications in the Social Sciences 43. (Sage Publications, Newbury Park, 1984).

25. Heumann, M., Uhlig, T. \& Zweck, J. True single domain and configurationassisted switching of submicron permalloy dots observed by electron holography. Phys. Rev. Lett. 94, 077202 (2005).

26. Hayashi, M., Thomas, L., Rettner, C., Moriya, R. \& Parkin, S. S. P. Direct observation of the coherent precession of magnetic domain walls propagating along permalloy nanowires. Nature Physics 3, 21-25 (2007).

27. Schryer, N. L. \& Walker, L. R. Motion of 180 Degrees Domain-Walls in Uniform Dc Magnetic-Fields. J. Appl. Phys. 45, 5406-5421 (1974).

28. Yang, J., Nistor, C., Beach, G. S. D. \& Erskine, J. L. Magnetic domain-wall velocity oscillations in permalloy nanowires. Phys. Rev. B 77 (2008).

29. Lee, J. Y., Lee, K. S., Choi, S., Guslienko, K. Y. \& Kim, S. K. Dynamic transformations of the internal structure of a moving domain wall in magnetic nanostripes. Phys. Rev. B 76 (2007).

30. Bryan, M. T., Schrefl, T. \& Allwood, D. A. Dependence of Transverse Domain Wall Dynamics on Permalloy Nanowire Dimensions. IEEE Trans. Magn. 46, 1135-1138 (2010).

31. Lewis, E. R., Petit, D., Jausovec, A. V., O’Brien, L., Read, D. E., Zeng, H. T. \& Cowburn, R. P. Measuring Domain Wall Fidelity Lengths Using a Chirality Filter. Phys. Rev. Lett. 102 (2009).

32. Nakatani, Y., Thiaville, A. \& Miltat, J. Faster magnetic walls in rough wires. Nat. Mater. 2, 521-523 (2003)

\section{Acknowledgements}

The ESPRC (grant no. EP/G004765/1; to WRB) and the Leverhulme Trust (grant no. F/ 07058/AW; to LFC) funded this scientific work. The US DoE under contract no. DE AC03 76 SF00098 funds the Advance Light Source. We are thankful for the resources provided by the National Academic Grid.

\section{Author contributions}

W.R.B. and L.F.C. conceived of the project. K.Z., S.L. and D.E.R. fabricated the samples. All authors planned, designed and oversaw the STXM experiments. K.Z., S.K.W., S.L. and D.E.R. executed the STXM at the Advanced light source with the technical support of T.T.; K.Z. and S.K.W. analysed the data and performed the magnetic simulations; K.Z., S.K.W. and W.R.B. drafted the paper.

\section{Additional information}

Supplementary information accompanies this paper at http://www.nature.com/ scientificreports

Competing financial interests: The authors declare no competing financial interests. License: This work is licensed under a Creative Commons Attribution 3.0 Unported License. To view a copy of this license, visit http://creativecommons.org/licenses/by/3.0/ How to cite this article: Zeissler, K. et al. The non-random walk of chiral magnetic charge carriers in artificial spin ice. Sci. Rep. 3, 1252; DOI:10.1038/srep01252 (2013). 